\title{
Modeling of rain attenuation and site diversity predictions for tropical regions
}

\author{
F. A. Semire ${ }^{1,2}$, R. Mohd-Mokhtar ${ }^{1}$, W. Ismail ${ }^{1}$, N. Mohamad ${ }^{1}$, and J. S. Mandeep ${ }^{3}$ \\ ${ }^{1}$ School of Electrical and Electronic Eng., Universiti Sains Malaysia, Engineering Campus, 14300, Nibong Tebal, Malaysia \\ ${ }^{2}$ Dept. of Electronic and Electrical Engineering, Ladoke Akintola University of Technology, P.M.B 4000, Ogbomoso, Nigeria \\ ${ }^{3}$ Dept. of Electrical, Electronic and System Engineering, Universiti Kebangsaan Malaysia, 43600, Bangi, Selangor, Malaysia
}

Correspondence to: F. A. Semire (fasemire@lautech.edu.ng)

Received: 19 March 2014 - Revised: 30 January 2015 - Accepted: 15 February 2015 - Published: 17 March 2015

\begin{abstract}
Presented in this paper is an empirical model for long-term rain attenuation prediction and statistical prediction of site diversity gain on a slant path. Rain attenuation prediction on a slant path is derived using data collected from tropical regions, and the formula proposed is based on Gaussian distribution. The proposed rain attenuation model shows a considerable reduction in prediction error in terms of standard deviation and root-mean-square (rms) error. The site diversity prediction model is derived as a function of site separation distance, frequency of operation, elevation angle and baseline orientation angle. The novelty of the model is the inclusion of low elevation angles and a high link frequency up to $70 \mathrm{GHz}$ in the model derivation. The results of comparison with Hodge, Panagopoulos and Nagaraja empirical predictions show that the proposed model provides a better performance for site separation distance and elevation angle. The overall performance of the proposed site diversity model is good, and the percentage error is within the allowable error limit approved by International Telecommunication Union Region (ITU-R).
\end{abstract}

Keywords. Meteorology and atmospheric dynamics (Climatology)

\section{Introduction}

In recent years, there has been a high demand for high data rates, wide bandwidths and high availability of satellite communication signals for multimedia services. Due to this great demand and overcongestion of the Ku-frequency band, satellite communication is now exploiting the $\mathrm{Ka}(20 / 30 \mathrm{GHz})$ band (Yeo et al., 2011; Luini et al., 2011; Pan et al., 2008).
However, microwave signals propagating in these bands suffer from more rain attenuation in comparison to the conventional $\mathrm{C}$ and $\mathrm{Ku}$ band. Therefore, in order to reduce the effect of attenuation on the communication links, several rain attenuation prediction models coupled with fade mitigation techniques have been proposed. The fade mitigation techniques include diversity protection schemes and power-control and adaptive-wave techniques (Castanet et al., 1998; Panagopoulos et al., 2004; ITU-R, 1994). Among the fade mitigation techniques, site diversity has been found to be the most efficient of all (Panagopoulos et al., 2004, 2005; ESA, 2002). The site diversity technique is based on the concept of the inhomogeneous nature of a rainfall event which occurs within a localized rain cell of a few kilometers in the horizontal and vertical extent (Panagopoulos et al., 2004; Hodge, 1982; Callaghan et al., 2008).

"Site diversity system" is a general term used in describing the utilization of two or more geographically separated Earth base stations in a satellite communication link to minimize the effect of attenuation due to rain during a exhaustive rainfall period. This concept is employed in such a way that if two Earth base stations are separated by at least the average horizontal extent of the rain cell, the cell may likely not intersect the satellite path of both ground stations at any given time (Ippolito, 2008). The signal streams received at each station are sent to the so-called reference station, where they are processed with certain criteria such as switching or selection or are combined so as to improve the signal-to-noise ratio, and a decision process is implemented to select the less attenuated signal for use in the communications system (Bosisio and Riva, 1998). 
The impact of site diversity on communication system performance is evaluated as a function of site diversity gain. Diversity gain is the improvement in the system margin at a given reliability level which results from the use of path diversity. Models for prediction of rain attenuation and site diversity performance are categorized into two major classes: (1) regression models based on long-term rain attenuation and site diversity statistics (Panagopoulos et al., 2005; Hodge, 1982; ITU-R, 2009; Moupfouma, 1984; Dissanayake et al., 1997; Mandeep et al., 2006) and (2) physical models based on the solution of wave equations for a medium with raindrops which employs lognormal distribution of rain rate and rain attenuation in prediction of joint probability of dual site diversity (Bosisio and Riva, 1998; Mass, 1987; Capsoni and Matricciani, 1985; Crane and Shieh, 1989; Matricciani, 1994).

This work presents rain attenuation and site diversity prediction models based on long-term beacon rain attenuation and both ground and satellite radar measurements. The two proposed models are derived from tropical region rainfall data and are easy to implement for rain attenuation prediction and site diversity performance evaluation.

This paper is arranged as follows: Sect. 2 describes the experimental setup and data collection, while Sect. 3 explains modeling of rain attenuation prediction using an appropriate regression fitting analysis. The derivation of this model is based on both radar and beacon measurements. The radar data are used for horizontal adjustment factor derivation, while a beacon measurement is used for vertical reduction factor formulation. The site diversity prediction model derived in line with the Hodge prediction model is presented in Sect. 4. The model data are derived from beacon measurement, which serves as a reference site, and Tropical Rainfall Measuring Mission (TRMM) data for diverse sites at different link parameters. The prediction capabilities of both models are tested against the ITU-R $618-10$ for rain attenuation prediction, and Hodge, Panagopoulos and Nagaraja models for site diversity gain prediction are shown in Sect. 5 (Panagopoulos, 2005; Hodge, 1982; ITU-R, 2009; Nagaraja and Otung, 2012). Finally, Sect. 6 draws some useful conclusions.

\section{Experimental setup and data collection}

Radar and beacon measurement techniques were employed in the derivation of the improved rain attenuation model. The receiver site for beacon measurement of signal attenuation due to rain was located at Universiti Sains Malaysia, USM $\left(5.17^{\circ} \mathrm{N}, 100.4^{\circ} \mathrm{E}\right)$. The Ku-band beacon receiver received signal from the SUPERBIRD-C satellite at $144^{\circ}$ at an elevation and azimuth angle of 40.1 and $95.4^{\circ}$, respectively. The antenna dish was set at $57 \mathrm{~m}$ above mean sea level. The output of the LNB (low-noise block) was connected to a data logger and interfaced with Kisyo software. Kisyo is the software that came with the beacon equipment. It is used for downloading and extracting signal information measured by the beacon. The signal was sampled at $1 \mathrm{~s}$ intervals and data were averaged over $1 \mathrm{~min}$. The data were collected for 5 years over the period of 2005 to 2009 . The average data availability was over $95 \%$.

The radar data employed in rain attenuation modeling were collected from the Malaysian Meteorological Department (MMD). The Malaysian radar system employs a 3D RAPIC system developed by the Australian Meteorological Bureau. The radar reflectivity data are converted to rainfall rate using the Marshall-Palmer radar reflectivity model (Marshall and Palmer, 1948). Four months of radar data from November 2008 to February 2009 were obtain from the MMD, from which only data from the Butterworth radar station were employed. The accuracy of the radar data as compared with rain gauge observation and data availability for the period of measurement were 92 and $98 \%$, respectively.

The modeling of site diversity gain prediction for South Asian countries was derived from spatial rainfall measurements obtained from TRMM Precipitation Radar (PR) data. The rain attenuation measurement was carried out at the following locations in the South Asia region: School of Electrical and Electronic Engineering, Universiti Sains Malaysia (USM), Institute of Technology Bandung (ITB) Indonesia; University of the South Pacific (USP), Fiji; King Mongkut's Institute of Technology Ladkrabang (KMITL), Thailand; and Anteneo de Manila University (AdMU), the Philippines. A reference site for each location was set at $\left(5.17^{\circ} \mathrm{N}, 100.4^{\circ} \mathrm{E}\right)$, $\left(6.5^{\circ} \mathrm{S}, 107.4^{\circ} \mathrm{E}\right),\left(18.1^{\circ} \mathrm{S}, 178.5^{\circ} \mathrm{E}\right),\left(13.7^{\circ} \mathrm{N}, 100.8^{\circ} \mathrm{E}\right)$ and $\left(14.7^{\circ} \mathrm{N}, 121.1^{\circ} \mathrm{E}\right)$ for USM, ITB, USP, KMITL and AdMU, respectively. The details of site and antenna specifications are shown in Table 1.

The system availability for the beacon data employed in site diversity modeling for the reference site was $93 \%$ for both 2002 and 2003. The site diversity stations were spaced at random from 1 to $50 \mathrm{~km}$ along a fixed baseline orientation spanning from 0 to $90^{\circ}$. The elevation angle and frequency of operation were varied from 10 to $50^{\circ}$ and 10 to $70 \mathrm{GHz}$, respectively. The corresponding diverse stations rainfall data between 2002 and 2003 were extracted from the TRMM website at a spatial and temporal resolution of $0.25^{\circ}$ by $0.25^{\circ}$ and monthly, respectively. The coarse spatial resolution was further interpolated linearly using the MATLAB platform by varying the location coordinate. The interpolated annual rainfall accumulation derived from the TRMM databank at specified locations was then converted to rain rate and consequently to rain attenuation in line with the proposed rain attenuation model for 100 hypothetical slant paths within the range of frequency and elevation angles shown in Table 2. The site diversity gain $G_{\mathrm{D}}(p)$ is modeled using Eq. (1):

$G_{\mathrm{D}}(p)=A_{\mathrm{S}}(p)-A_{\mathrm{J}}(p)$, 
Table 1. Site and antenna specifications.

\begin{tabular}{lccccc}
\hline Location & USM & KMITL & ITB & USP & AdMU \\
\hline Earth station location & $5.17^{\circ} \mathrm{N}$ & $13.7^{\circ} \mathrm{N}$ & $6.5^{\circ} \mathrm{S}$ & $18.1^{\circ} \mathrm{S}$ & $14.7^{\circ} \mathrm{N}$ \\
& $100.4^{\circ} \mathrm{E}$ & $100.8^{\circ} \mathrm{E}$ & $107.4^{\circ} \mathrm{E}$ & $178.5^{\circ} \mathrm{E}$ & $121.1^{\circ} \mathrm{E}$ \\
Beacon frequency $(\mathrm{GHz})$ & 12.255 & 12.74 & 12.247 & 12.255 & 12.255 \\
Downlink polarization & & & Horizontal & & \\
Antenna diameter (m) & 2.4 & 2.4 & 1.8 & 1.8 & 1.8 \\
Antenna receiving gain (dBi) & 47.9 & 48.3 & 45.4 & 45.7 & 45.7 \\
\hline
\end{tabular}

where $A_{\mathrm{S}}(p)$ and $A_{\mathrm{J}}(p)$ are the single-site and joint attenuation values at probability $p$, respectively.

$$
A_{\mathrm{J}}(p)=\min \left\{A_{1}(p), A_{2}(p)\right\},
$$

where $A_{1}(p)$ and $A_{2}(p)$ are the instantaneous rain attenuation values at the reference and diversity station, respectively.

\section{Proposed rain attenuation model}

The proposed model is derived in two phases: the horizontal and vertical reduction factors. These factors are included so as to accommodate the impact of the inhomogeneous nature of rainfall events on the prediction model. The horizontal reduction factor of the model is derived based on the Goddard concept, with an assumption that there is a virtual link along every radial line, which can be likened to a transmission path on which a signal can be propagated (Goddard and Thurai, 1997). The horizontal adjustment factor is deduced from rain rate, and derived rain attenuation is extracted from Malaysian radar rainfall data at a radar line of $7 \mathrm{~km}$ from the referenced site. Regarding the vertical reduction factor, the effect of horizontal adjustment is normalized from the 5-year slant path attenuation measurements at USM and the derived parameter is used in linear regression fitting for deduction of the vertical reduction factor. The regression fitting of attenuation against the vertical reduction factor follows Gaussian distribution. The proposed attenuation model is given as follows.

The vertical and reduction factors $r_{\mathrm{V}}$ and $r_{\mathrm{h}}$ are given as

$r_{\mathrm{v}_{0.01}}=1.429 \exp \left(-\left(\frac{\frac{1}{\gamma_{R_{0.01}} L_{\mathrm{s}}}-0.0251}{0.0108}\right)^{2}\right)$,

$r_{\mathrm{h}_{0.01}}=a L_{\mathrm{g}}^{b}+c$,

with

$a=2.989 \exp \left(-\left(\frac{f-45.83}{23.61}\right)^{2}\right)$,

$b=-8128 f^{-3.329}-0.1432$, $c=-0.00006553 f^{3}-0.005906 f^{2}+0.08657 f+0.1513$,

$L_{\mathrm{g}}=L_{\mathrm{s}} \cos (\theta)$,

$L_{\mathrm{S}}=\frac{H_{\mathrm{R}}-H_{\mathrm{S}}}{\sin (\theta)}$,

where $f$ is the frequency of operation, $\theta$ is the elevation angle, $H_{\mathrm{R}}$ is the rain height and $H_{\mathrm{S}}$ is the altitude of the Earth station. The path attenuation exceeded for $0.01 \%$ percent of the time is

$A_{0.01}=\gamma_{\mathrm{R}_{0.01}} L_{\mathrm{s}} r_{\mathrm{v}_{0.01}} r_{\mathrm{h}_{0.01}}$.

Attenuation at other time percentage $(p)$ ranging from 0.001 to $1 \%$ is

$\frac{A_{\mathrm{p}}}{A_{0.01}}=0.117 p^{-(0.637+0.0371 \log (p))}$.

The agreement of the proposed model is tested using error prediction algorithm (ITU-R, 2009b). The procedure is as follows:

- estimate the ratio $S_{i}$ of the predicted attenuation $A_{S_{i}}$ to the measured attenuation $A_{\mathrm{m}_{i}}$ for each radio link

$$
S_{i}=\frac{A_{\mathrm{S}_{i}}}{A_{\mathrm{m}_{i}}}
$$

- calculate the corresponding test variable as

$$
V_{i}=\left\{\begin{array}{l}
\ln S_{i}, \quad \text { for } A_{m_{i}} \geq 10 \mathrm{~dB} \\
\left(\frac{A_{m_{i}}}{10}\right)^{0.2} \ln S_{i}, \quad \text { for } A_{m_{i}} \prec 10 \mathrm{~dB}
\end{array}\right\}
$$

- repeat the procedure for each time percentage 
Table 2. Frequency and elevation angle of the diverse stations.

\begin{tabular}{cccccccccc}
\hline $\begin{array}{c}\text { Freq. } \\
(\mathrm{GHz})\end{array}$ & $\begin{array}{c}\text { Elev. } \\
\left(\theta^{\circ}\right)\end{array}$ & $\begin{array}{c}\text { Freq. } \\
(\mathrm{GHz})\end{array}$ & $\begin{array}{c}\text { Elev. } \\
\left(\theta^{\circ}\right)\end{array}$ & $\begin{array}{c}\text { Freq. } \\
(\mathrm{GHz})\end{array}$ & $\begin{array}{c}\text { Elev. } \\
\left(\theta^{\circ}\right)\end{array}$ & $\begin{array}{c}\text { Freq. } \\
(\mathrm{GHz})\end{array}$ & $\begin{array}{c}\text { Elev. } \\
\left(\theta^{\circ}\right)\end{array}$ & $\begin{array}{c}\text { Freq. } \\
(\mathrm{GHz})\end{array}$ & $\begin{array}{c}\text { Elev. } \\
\left(\theta^{\circ}\right)\end{array}$ \\
\hline 12.255 & 10 & 12.255 & 20 & 12.255 & 30 & 12.255 & 40 & 12.255 & 50 \\
20 & 10 & 20 & 20 & 20 & 30 & 20 & 40 & 20 & 50 \\
50 & 10 & 50 & 20 & 50 & 30 & 50 & 40 & 50 & 50 \\
70 & 10 & 70 & 20 & 70 & 30 & 70 & 40 & 70 & 50 \\
\hline
\end{tabular}

- calculate the mean $\mu_{\mathrm{V}}$, the standard deviation $\sigma_{\mathrm{V}}$, and the rms value $\rho_{\mathrm{V}}$ of the $V_{i}$ values for each time percentage;

$$
\rho_{\mathrm{V}}=\left(\mu_{\mathrm{V}}^{2}+\sigma_{\mathrm{V}}^{2}\right)^{0.5}
$$

The cumulative distribution of the measured rain attenuation is compared with three existing prediction models. The comparison result is shown in Fig. 1a and b. Figure 1a shows that the Garcia model (Garcia and da Silvo Mello, 2004) underestimates the measured rain attenuation values at every percentage of time. The ITU-R model underestimates at 1 to $0.8 \%$ and 0.08 to $0.001 \%$ of availability time and overestimates at 0.3 to $0.01 \%$. The same trend is observed in the Goddard and Thurai model. The proposed model agrees reasonably well with measured values from low availability time up to $0.008 \%$ time percentage. The deviation of the proposed model from the experimental values at time percentage above $0.008 \%$ may be due to saturation of rainfall at a high rain rate (Mandeep and Allnutt, 2007).

The results of error, standard deviation and rms error values of the proposed and three other existing models are shown in Figs. 1b and 2a, b. The ITU-R and Goddard and Thurai models exhibit a similar behavior. The highest mean error is observed in the Garcia model. The proposed model shows a significant improvement over the existing prediction models, with considerable reduction in rms error as compared with other testing models.

\section{Proposed site diversity model}

The site diversity prediction model is derived based on rain attenuation measurements from five countries in the South Asia region. A regression fitting is obtained on the numerical results obtained from rain attenuation and site diversity measurements. The dependence of diversity gain on four major link factors (site separation distance $D$, common elevation angle of slant path $B$, link frequency $f$, and orientation of the baseline between the two Earth stations $\beta$ ) is modeled.

In the deduction of the model of site separation distance dependence, five sets of data are selected for each location at different separation distances with a frequency of operation at $12.255 \mathrm{GHz}$ for USM, AdMU and USP; $12.74 \mathrm{GHz}$
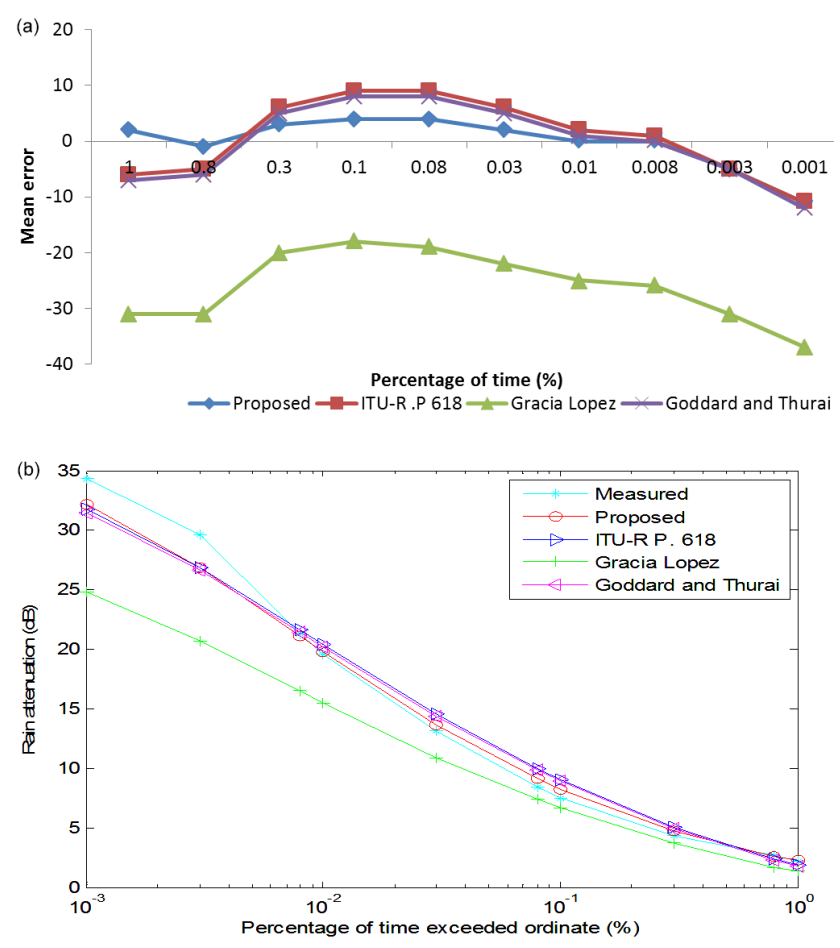

Figure 1. (a) Comparison of measured and predicted rain attenuation distribution. (b) Results of comparative test of models (mean).

for KMITL; and $12.247 \mathrm{GHz}$ for ITB. The elevation angle and baseline orientation angle are kept at 30 and $0^{\circ}$, respectively. The regression fit of diversity gain as a function of $D$ is shown in Fig. 3. Further regression analysis is performed to determine the set of equations that define the two coefficients $a$ and $b$. Single-site attenuation is plotted against coefficient $a$ and $b$, and the resulting regression curves with their corresponding equations are shown in Fig. 4.

The dependence of frequency of operation is modeled by selecting rain attenuation measurements at different frequencies ranging from 10 to $70 \mathrm{GHz}$ from the database, while keeping separation distance, elevation angle and baseline angle at $11 \mathrm{~km}$, is $30^{\circ}$ and $0^{\circ}$, respectively. The set of data selected for frequency dependence is normalized to remove its dependence on separation distance, $D$. The resulted normalized $G_{\mathrm{f}}$ is plotted against frequency of operation, and the resulting regression fit of diversity gain as a function of fre- 

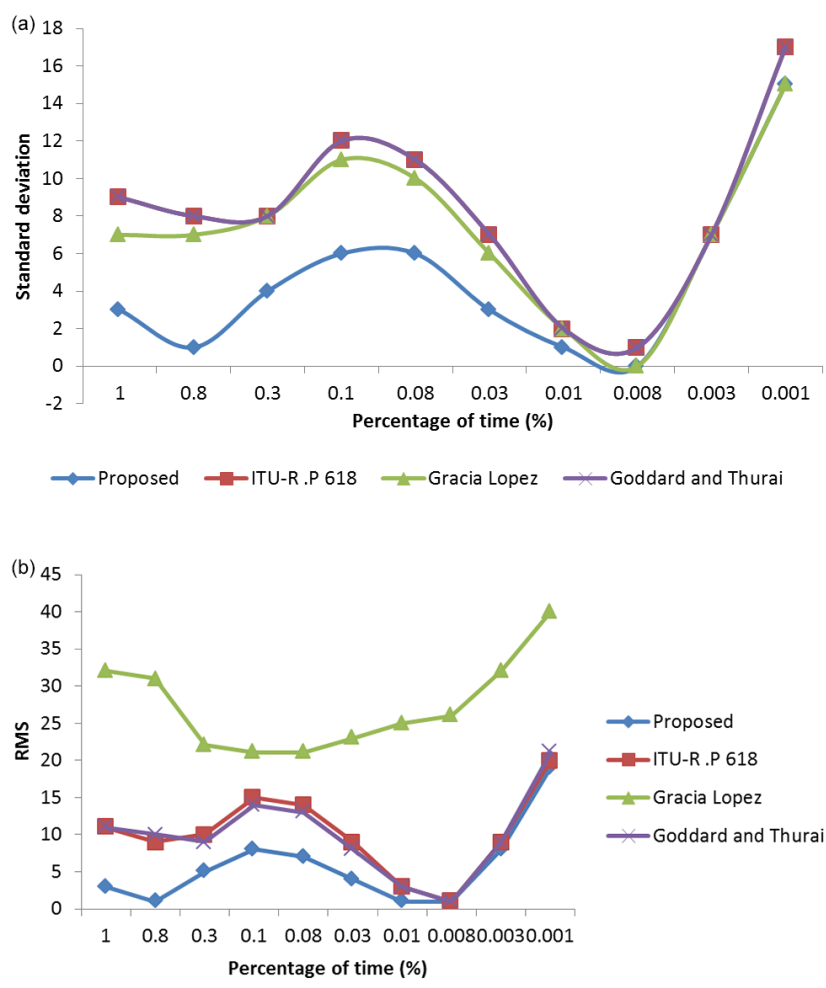

Figure 2. (a) Results of comparative test of models (standard deviation). (b) Results of comparative test of models (rms).

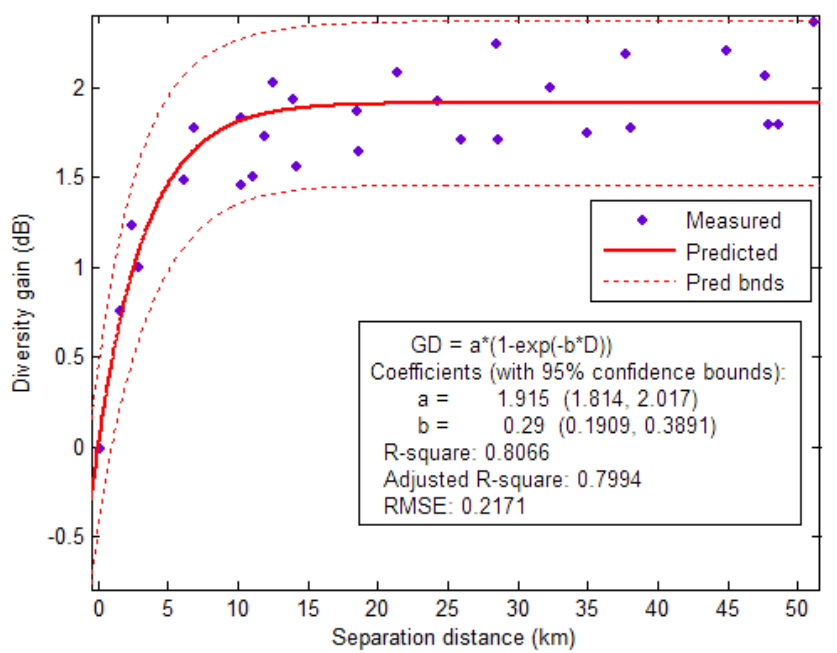

Figure 3. The nonlinear regression curve between diversity gain and separation distance.

quency $f$ along with the rms error is shown in Fig. 5 for the USM and USP stations. Similar results are derived for the three other stations, and the coefficient of the regression equation is shown in Table 3.

The dependence of elevation angle is also determined by following the same procedure as used for frequency dependence. The dependence of elevation on the attenuation mea- (a)

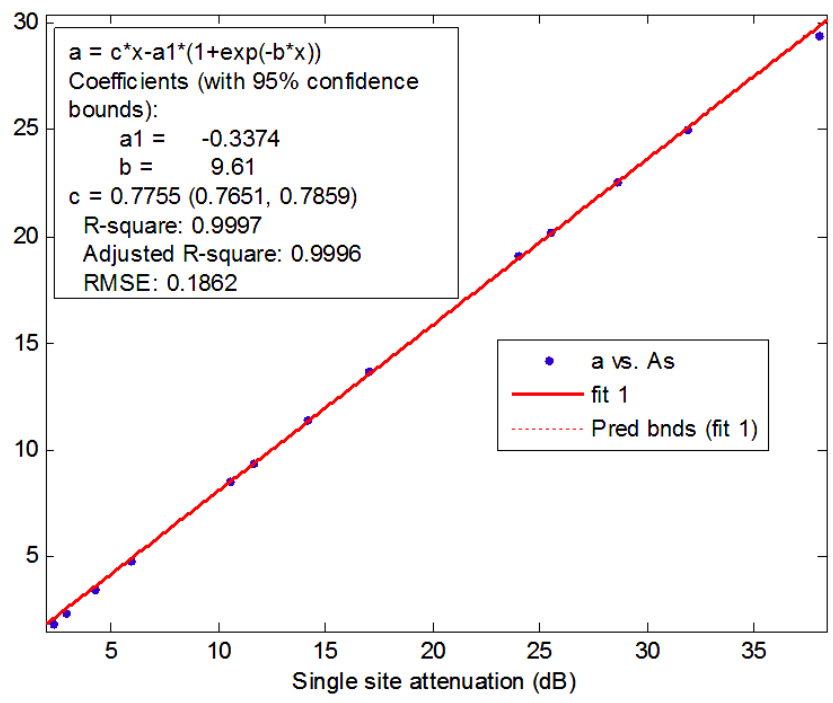

(b)

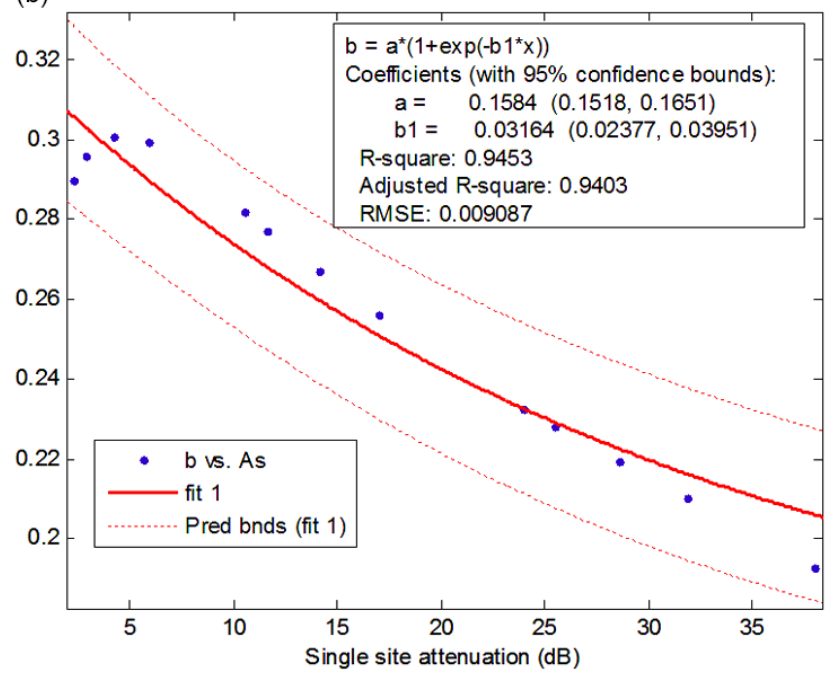

Figure 4. (a) Regression line of fit for coefficient $a$. (b) Regression line of fit for coefficient $b$.

surement is estimated by extracting values of rain attenuation at different elevation angles from 10 to $50^{\circ}$ while keeping other link parameters constant. The set of values derived are also normalized to remove the effect of site separation and frequency. The regression plot of elevation angle dependence follows a nonlinear quadratic law model as shown in Fig. 6, and the constants of the coefficient for all the five stations are shown in Table 4.

The dependence of diversity gain on the orientation of the baseline relative to the propagation path is also examined. The regression fit obtained is shown in Fig. 7, and the corresponding coefficient constants are shown in Table 5.

The model expression is given as

$G(D, f, B, \beta)=G_{D} G_{f} G_{B} G_{\beta}$, 

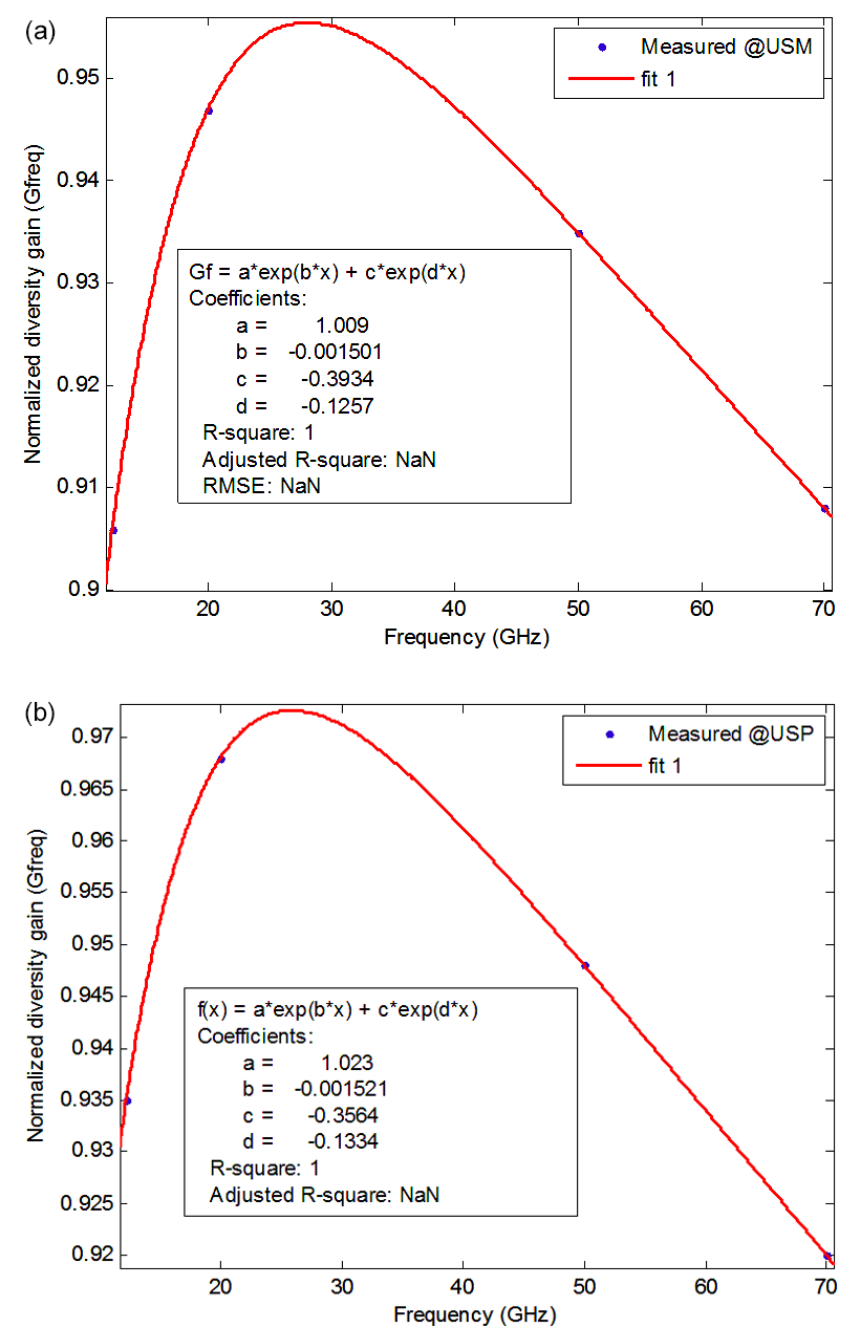

Figure 5. (a) Nonlinear regression fit of diversity gain as a function of frequency (USM). (b) Nonlinear regression fit of diversity gain as a function of frequency (USP).

with

$G_{D}=a\left(1-e^{-b D}\right)$,

$a=0.7755 A_{\mathrm{S}}+0.3374\left(1+\exp ^{-9.16 A_{\mathrm{S}}}\right)$,

$b=0.1584\left(1+\exp ^{-0.03164 A_{\mathrm{S}}}\right)$,

$G_{f}=1.006 \mathrm{exp}^{-0.0015 f}-0.395 \exp ^{-0.473 f}$,

$G_{B}=0.899\left(1+B^{-0.683}\right)$,
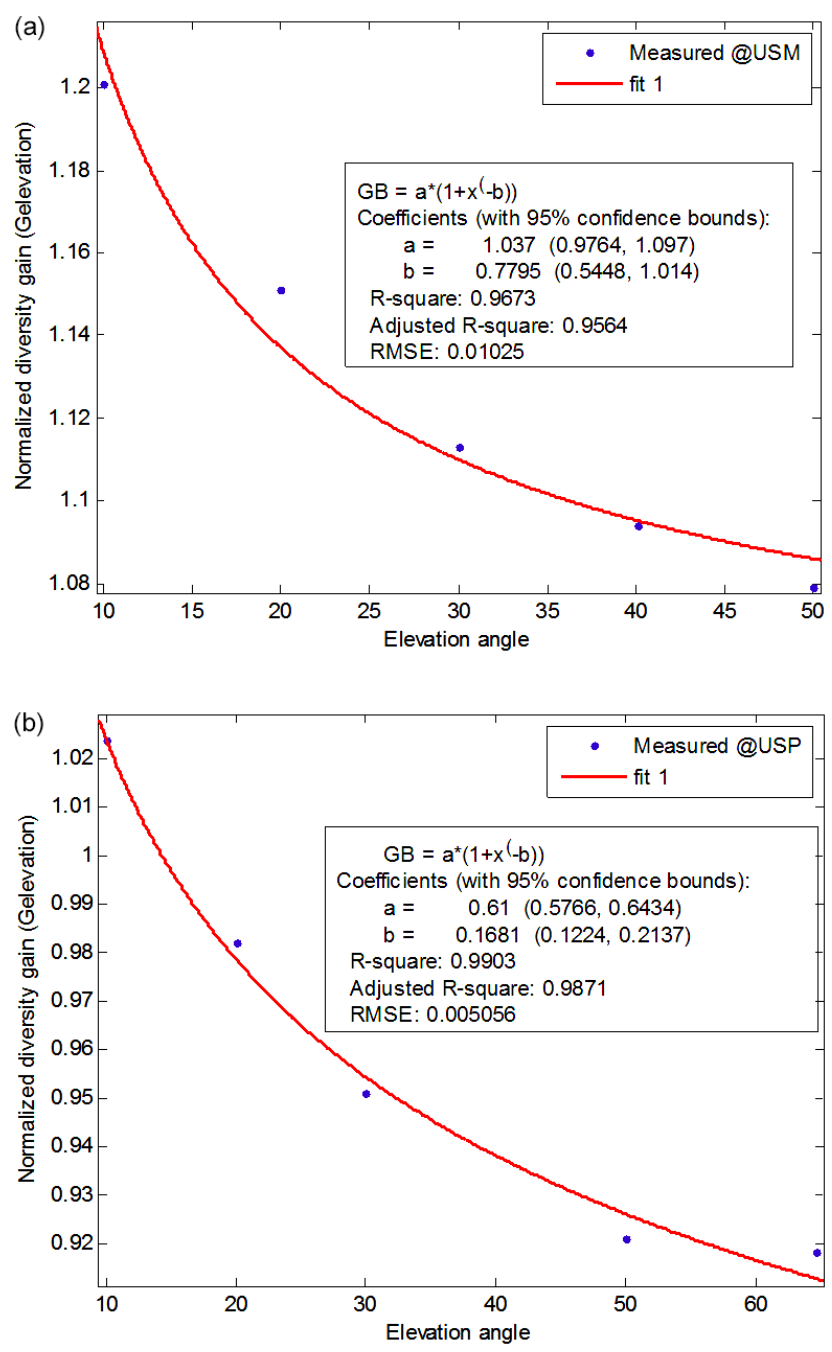

Figure 6. (a) Nonlinear regression fit of diversity gain as a function of elevation angle (USM). (b) Nonlinear regression fit of diversity gain as a function of elevation angle (USP).

$G_{\beta}=-0.0000015 \beta+0.9877$,

where $D$ is the separation distance in kilometers, $A_{\mathrm{S}}$ is the single-site attenuation in decibels, $f$ is the link frequency in gigaherz, $B$ is the angle of elevation in degrees and $\beta$ is the baseline orientation angle in degrees. The modeling procedure is in line with the Hodge prediction model, but different expressions and coefficients are obtained for tropical regions.

\section{Performance evaluation of the prediction models}

The agreement of the proposed model with experimental results is tested using the concept of relative diversity gain $g$ as 

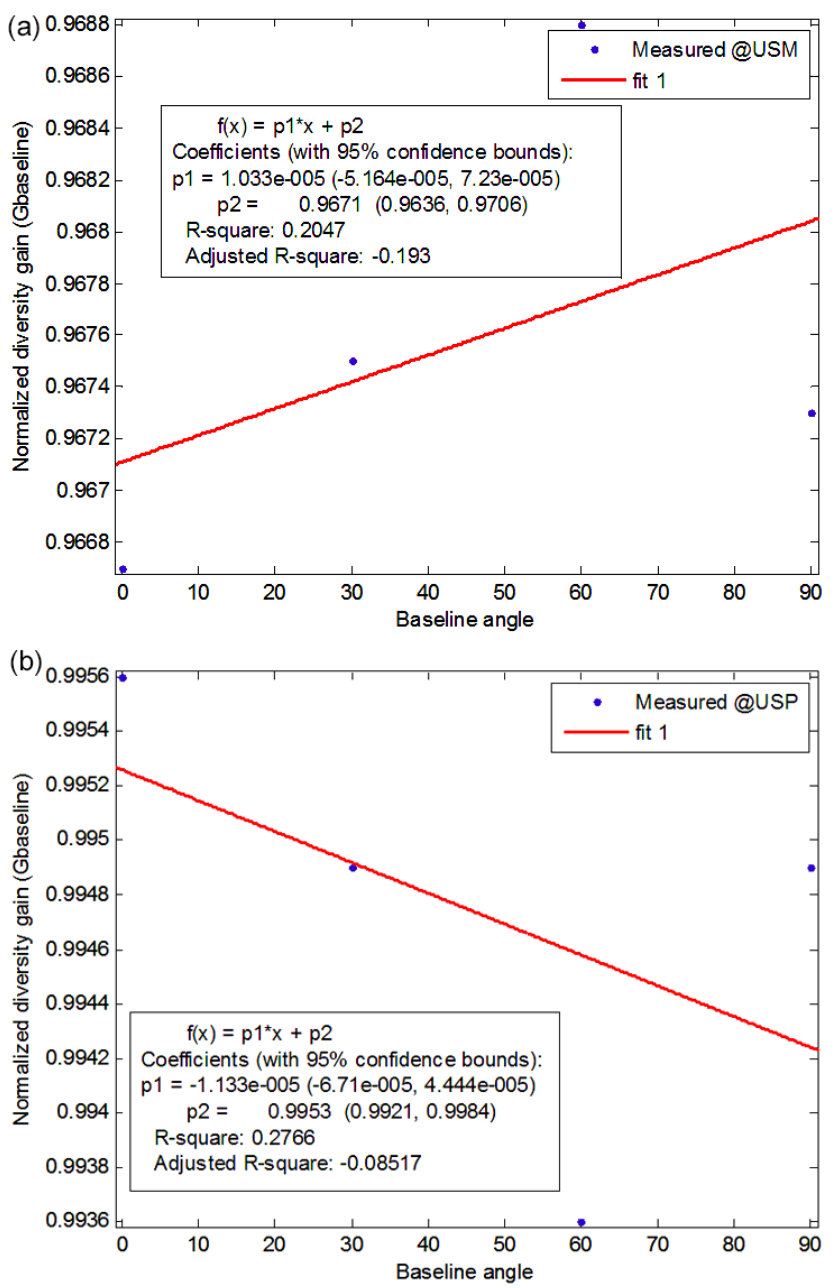

Figure 7. (a) Nonlinear regression fit of diversity gain as a function of baseline orientation angle (USM). (b) Nonlinear regression fit of diversity gain as a function of baseline orientation angle (USP).

Table 3. Constants of regression coefficient for operating frequency dependence.

\begin{tabular}{lcccc}
\hline & $\mathrm{a}$ & $\mathrm{b}$ & $\mathrm{c}$ & $\mathrm{d}$ \\
\hline USM & 1.009 & -0.0015 & -0.3934 & -0.1257 \\
KMITL & 1.007 & -0.00131 & -0.3653 & -0.09802 \\
ITB & 1.003 & -0.00185 & $-1.70 \times 10^{8}$ & -1.858 \\
USP & 1.023 & -0.00152 & -0.3564 & -0.1334 \\
AdMu & 0.9878 & -0.00133 & -0.4656 & -0.1485 \\
Average & 1.00596 & -0.0015 & -0.39518 & -0.47272 \\
\hline
\end{tabular}

described below:

$g=\frac{G}{A_{\mathrm{S}}}$

The parameter $g$ is less dependent on attenuation than $G$. The percentage error $\varepsilon$ is defined as

$\varepsilon(p)=100\left[g_{\text {est }}(p)-g_{\text {mea }}(p)\right]$,
Table 4. Constants of regression coefficient for elevation angle dependence.

\begin{tabular}{lcc}
\hline & $\mathrm{a}$ & $\mathrm{b}$ \\
\hline USM & 1.037 & 0.7795 \\
KMITL & 0.9444 & 0.7479 \\
ITB & 0.61 & 0.1681 \\
USP & 0.9803 & 0.9727 \\
AdMu & 0.9241 & 0.7481 \\
Average & 0.89916 & 0.68326 \\
\hline
\end{tabular}

Table 5. Constants of regression coefficient for elevation angle dependence.

\begin{tabular}{lcc}
\hline & $\mathrm{p} 1$ & $\mathrm{p} 2$ \\
\hline USM & $1.03 \times 10^{-5}$ & 0.9671 \\
KMITL & $1.03 \times 10^{-5}$ & 0.9671 \\
ITB & $-1.90 \times 10^{-5}$ & 1.005 \\
USP & $-1.13 \times 10^{-5}$ & 0.9953 \\
AdMu & $2.00 \times 10^{-6}$ & 1.004 \\
Average & $-1.5 \times 10^{-6}$ & 0.9877 \\
\hline
\end{tabular}

where $g_{\text {est }}(p)$ and $g_{\text {mea }}(p)$ are, respectively, the predicted and measured relative gains for a given probability (Bosisio and Riva, 1998; Matricciani, 1994).

The model is tested along with some other existing site diversity gain prediction models like Hodge, Panagopoulos and Nagaraja. The rms error is determined as the difference between predicted and measured gain for the 13 time percentage values from 0.001 to $1.0 \%$. The equation is given as

$\mathrm{rms}={\sqrt{\frac{\sum\left(\frac{G_{\text {pred }}-G_{\text {meas }}}{G_{\text {meas }}}\right)}{13}}}_{2}$.

The proposed model performance is compared with site diversity measurement and prediction estimates provided by the Panagopoulos and Nagaraja models. The performance of the models as a function of distance is shown in Fig. 8, with other site parameters set at $12.255 \mathrm{GHz}$ for link frequency, $30^{\circ}$ for elevation angle and $0^{\circ}$ for baseline orientation angle. The results depicted in the figures show that the proposed model performed better than the existing models, which are largely derived from temperate regions. The site diversity gain prediction for $24.22 \mathrm{~km}$ at USM is closer to the experimental measurement compared to site diversity (SD) gain at $11.82 \mathrm{~km}$. The results at KMITL and AdMu show similar trends to that of USM. Diversity gain at USP and ITB shows a slightly high deviation from the experimental value. However, the difference is still incomparable with the deviation recorded for other existing models. This shows that, despite the difference in the experimental values and the predicted one, the model's performance still makes it preferable 

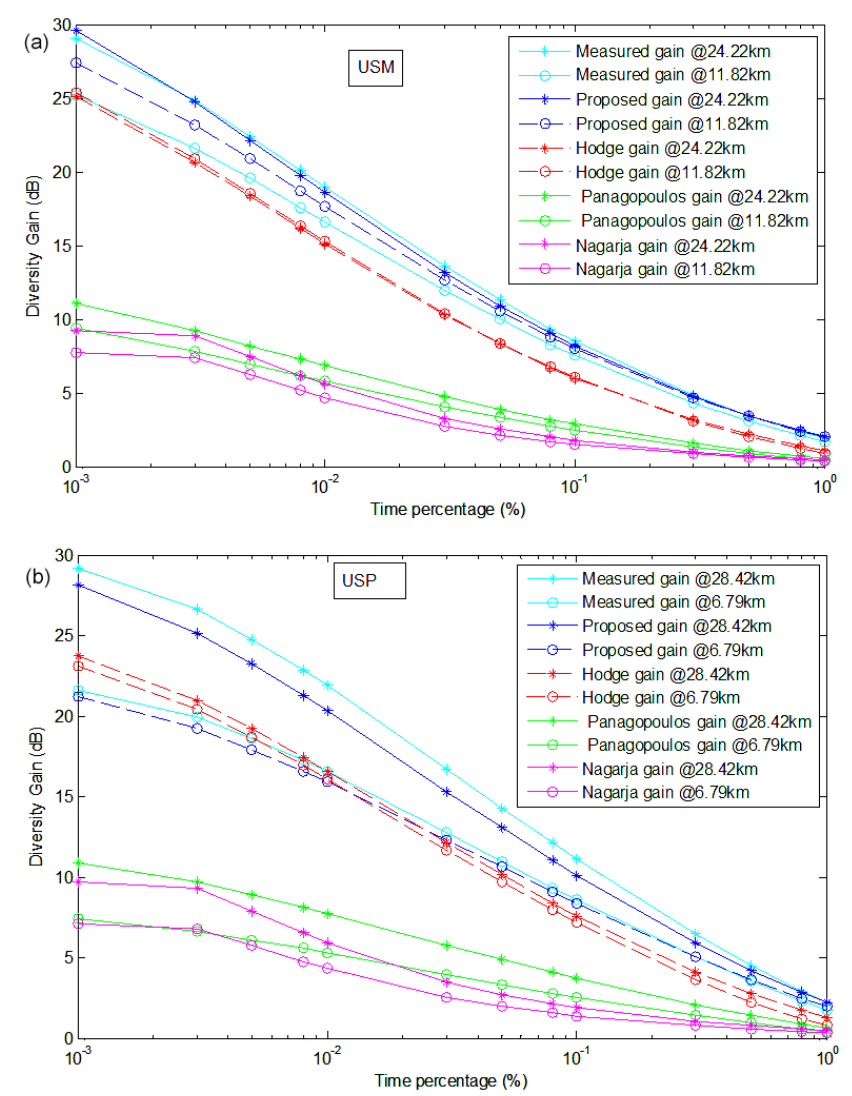

Figure 8. (a) Cumulative distribution of diversity gain predictions at different site separation distances (USM). (b) Cumulative distribution of diversity gain predictions at different site separation distances (USP).

to other existing models. The details of the performance analysis in terms of mean error and root mean square at $0.01 \%$ time percentage are given in Table 6.

The proposed model presents a reduced relative diversity mean error of $4.12,1.36,0.67,-2.50$ and $3.41 \%$ for USM, KMITL, ITB, USP and AdMU, respectively, at $0.01 \%$ time percentage. The rms ranges from 5 to $9.10 \%$. The performance degrades slowly with increasing distance. The Hodge model has relative diversity mean errors from -1.70 to $10.39 \%$ at $0.01 \%$ time percentage, with a corresponding rms not more than $30 \%$. The Panagopoulos model presents mean error slightly above $40 \%$ and $\mathrm{rms}$ around $70 \%$. The Nagaraja model has a similar trend for percentage mean error and rms of above 50 and $70 \%$, respectively. The details are shown in Table 6.

The performance of the model as a function of operating frequency is shown in Fig. 9. With site parameters kept at $10 \mathrm{~km}$ site separation, $30^{\circ}$ elevation angle and $0^{\circ}$ of baseline orientation for all the five locations, link frequency is varied between 12.255 and $20 \mathrm{GHz}$. The cumulative distribution of site diversity gain prediction for $20 \mathrm{GHz}$ at USM is closer to the experimental measurement compared to SD
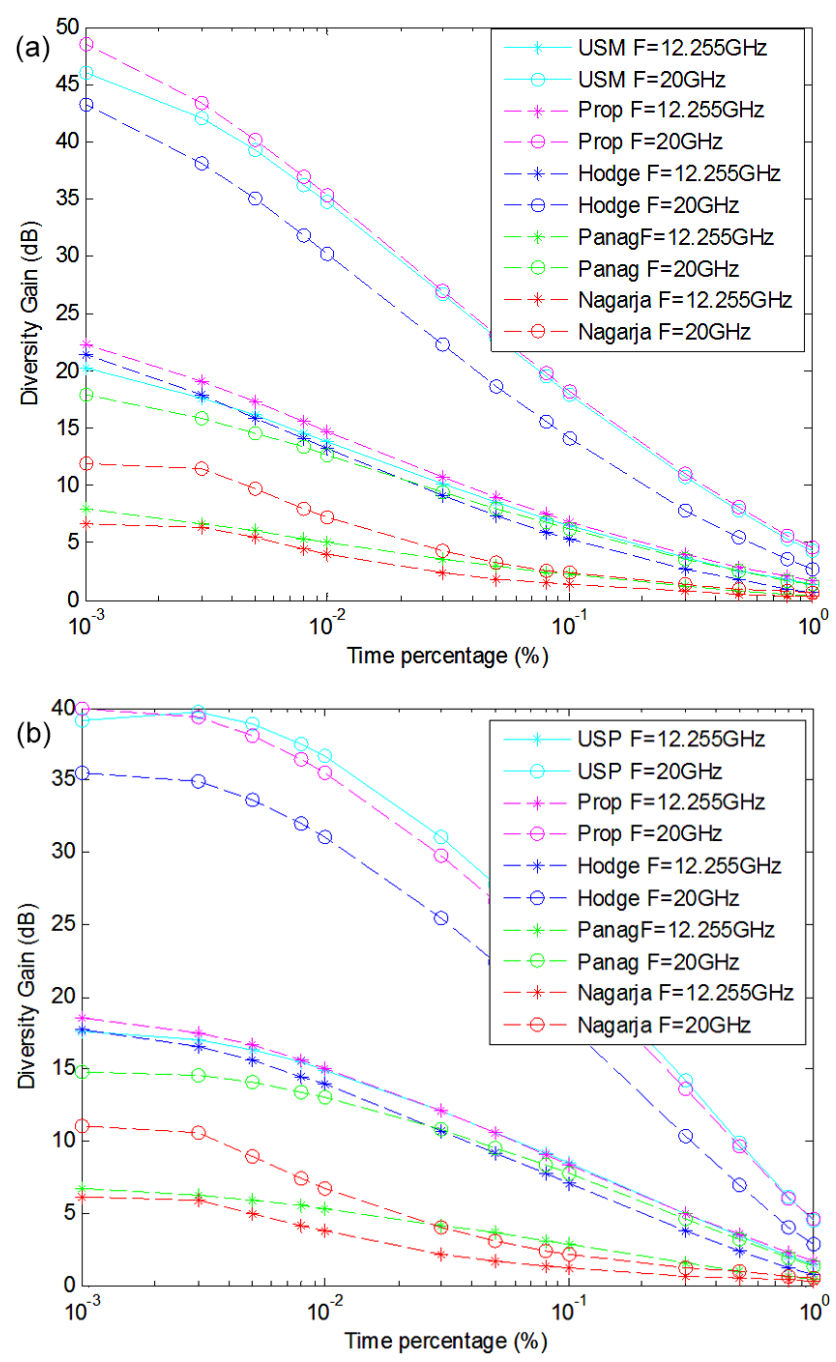

Figure 9. (a) Cumulative distribution of diversity gain predictions at different frequency of operation (USM). (b) Cumulative distribution of diversity gain predictions at different frequency of operation (USP).

gain at $12.255 \mathrm{GHz}$. The results at KMITL, ITB, USP and AdMU show similar trends to that of USM. Diversity gain predicted by Hodge at all locations shows a slight deviation from the experimental value. The two other models predict very high deviation from the experimental values. The reasons for the discrepancies can be traced back to regional differences. However, the Hodge model prediction is closer to the measured values because some of the data used in the derivation of its model are at Ku-band frequency.

The performance analysis in terms of mean error and root mean square for the proposed model presents a reduced percentage relative diversity mean error of $1.12,-1.83$, $-2.85,-2.04$ and $-0.98 \%$ for USM, KMITL, ITB, USP and AdMU, respectively, at $20 \mathrm{GHz}$ at $0.01 \%$ time percentage. The rms ranges from 1.85 to $3.557 \%$. The Hodge model has relative diversity mean errors from -6.54 to $-9.66 \%$ 
Table 6. Performance evaluation of site diversity gain prediction models at $0.01 \%$ time percentage.

\begin{tabular}{|c|c|c|c|c|c|c|c|c|}
\hline & \multicolumn{4}{|c|}{ Mean error $10 \mathrm{~km}(\%)$} & \multicolumn{4}{|c|}{ rms $10 \mathrm{~km}(\%)$} \\
\hline & Proposed & Hodge & Panagopoulos & Nagaraja & Proposed & Hodge & Panagopoulos & Nagaraja \\
\hline USM & 4.12 & -5.45 & -45.15 & -52.57 & 9.10 & 23.03 & 66.97 & 74.90 \\
\hline KMITL & 1.36 & -8.31 & -45.56 & -45.41 & 6.74 & 29.26 & 69.37 & 70.14 \\
\hline ITB & 0.67 & -10.39 & -47.53 & -53.19 & 5.27 & 24.28 & 68.48 & 76.97 \\
\hline USP & -2.50 & -1.70 & -42.02 & -45.79 & 3.89 & 23.97 & 68.05 & 78.55 \\
\hline \multirow[t]{3}{*}{ AdMU } & 3.41 & -6.52 & -44.15 & -45.89 & 8.72 & 26.57 & 68.13 & 71.52 \\
\hline & \multicolumn{4}{|c|}{ Mean error $20 \mathrm{~km}(\%)$} & \multicolumn{4}{|c|}{ rms $20 \mathrm{~km}(\%)$} \\
\hline & Proposed & Hodge & Panagopoulos & Nagaraja & Proposed & Hodge & Panagopoulos & Nagaraja \\
\hline USM & -1.49 & -15.75 & -49.21 & -54.28 & 2.99 & 27.92 & 65.76 & 73.64 \\
\hline KMITL & -5.54 & -21.65 & -52.77 & -52.40 & 5.95 & 33.14 & 66.87 & 67.67 \\
\hline ITB & -8.22 & -22.48 & -53.39 & -58.31 & 8.92 & 32.55 & 65.25 & 73.09 \\
\hline USP & -5.83 & -19.69 & -52.56 & -59.42 & 6.76 & 30.41 & 66.15 & 76.45 \\
\hline AdMU & -2.80 & -17.97 & -50.44 & -52.44 & 3.83 & 30.21 & 66.28 & 69.80 \\
\hline
\end{tabular}

at $0.01 \%$ time percentage for all locations, with a corresponding rms not more than $25 \%$. The Panagopoulos model presents mean error slightly above $-40 \%$ and $\mathrm{rms}$ around $65 \%$. The Nagaraja model has similar trend for percentage mean error and rms of above -50 and $80 \%$, respectively.

The performance of the model as a function of elevation angle is also examined, with site parameters kept at $10 \mathrm{~km}$ site separation, $12 \mathrm{GHz}$ and $0^{\circ}$ of baseline orientation for all the five locations. The dish elevation was varied between 10 and $30^{\circ}$. The cumulative distribution of site diversity gain prediction for all stations at $10^{\circ}$ is closer to the experimental measurement compared to SD gain at an elevation angle of $30^{\circ}$. The results of the cumulative distribution function of site diversity at an elevation of $30^{\circ}$ are much closer to the Hodge prediction model for all stations. The closeness of the Hodge model at an elevation of $30^{\circ}$ is due to the database used for the derivation of the model. The database used is composed of measurements taken at $30^{\circ}$ and above. The prediction error of the Hodge model at an elevation angle of $10^{\circ}$ is high. Therefore, the Hodge model cannot predict well for site diversity gain at a low elevation angle. The two other existing models follow the same trend of high deviations in site diversity gain from the experimental values. Although the elevation angle used in the derivation of the model is within the measured elevation angle range, the thresholds of data in the models are based on the data from temperate regions. Thus, the Panagopoulos and Nagaraja models tend to underestimate the diversity gain in tropical regions. The details are shown in Fig. 10.

The cumulative distribution of site diversity gain prediction at both 30 and $60^{\circ}$ baseline orientation angle at $10 \mathrm{~km}$ site separation, $30^{\circ}$ elevation angle and $12 \mathrm{GHz}$ frequency are closely related at all stations. It is observed that the Hodge model also has a similar pattern to the measured and proposed model. It is apparent that baseline angle has no or little correlation between baseline angle and site diversity gain. As is evident in Fig. 11, the thresholds of both orientation angles are almost the same. This implies that there are insignificant changes in the cumulative distribution function of site diversity gain with respect to increase in orientation angle. The observed trends for the other two existing models follow the same pattern, with little or no changes in the observed threshold as baseline angle increases. Although the Panagopoulos and Nagaraja models follow the same trend to that of the experimental value, they both tend to underestimate site diversity gain in the region.

The performance analysis of the proposed model presents a reduced percentage relative diversity error of $2.05,1.51$, $0.43,-0.27$ and $2.45 \%$ for USM, KMITL, ITB, USP and AdMU, respectively, at $60^{\circ}$ baseline angle at $0.01 \%$ time percentage. The lowest and highest percentage error is observed at ITB and AdMU, respectively. The estimated rms is below $10 \%$. The Hodge model has relative diversity mean errors between 2.29 and $11.25 \%$ at $0.01 \%$ time percentage for all locations, with a corresponding rms less than $30 \%$. The Panagopoulos model presents mean error slightly above $-40 \%$, and rms is around $60 \%$. The Nagaraja model has a similar trend for percentage mean error and rms of above -50 and $70 \%$, respectively.

\section{Conclusion}

Two new prediction models have been proposed. The proposed model for long-term rain attenuation prediction on a slant path is derived based on data collected from tropical regions and the formula proposed follows a Gaussian distribution function. The performance of the proposed model is tested using three existing prediction models, and the results are very encouraging. The model proposed presents 

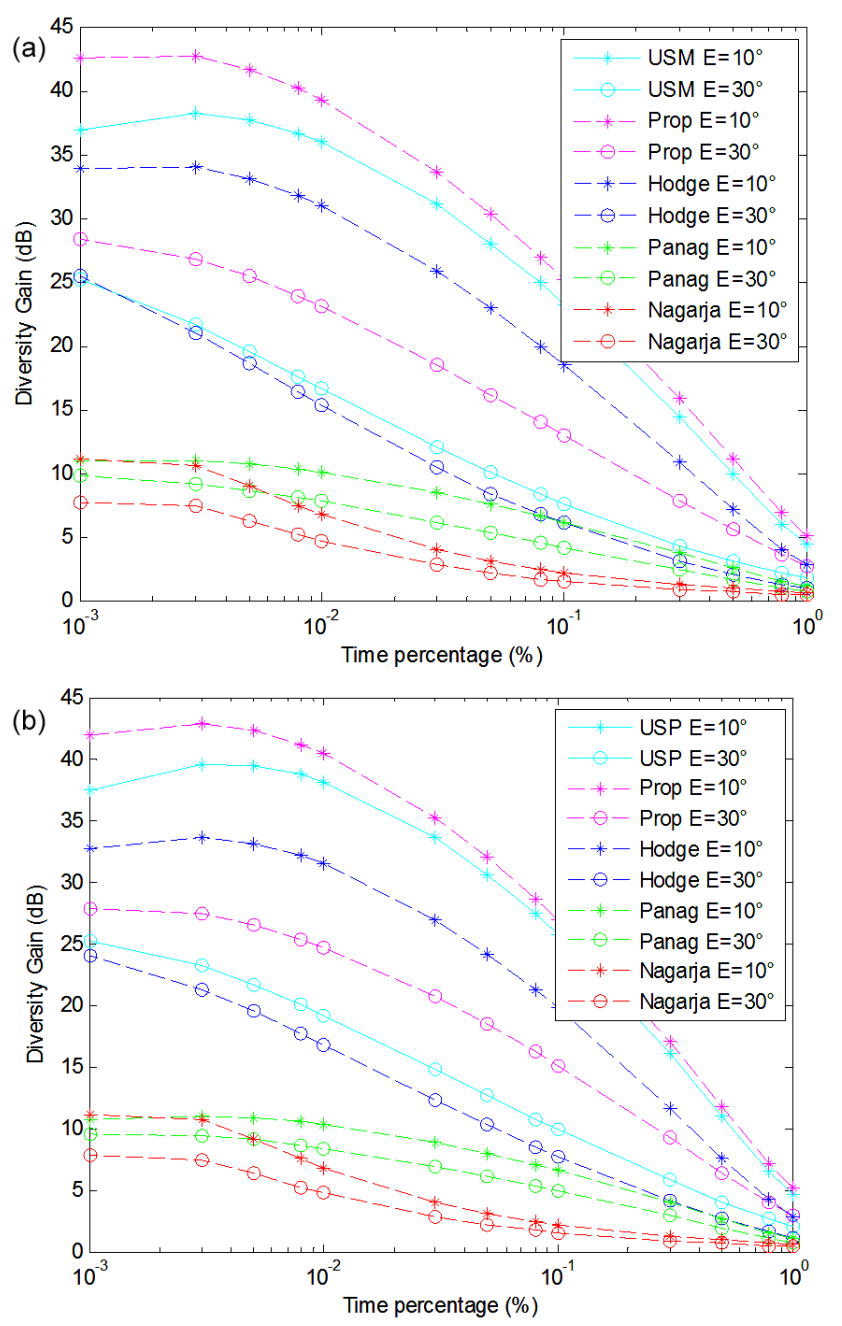

Figure 10. (a) Cumulative distribution of diversity gain predictions at different elevation angle (USM). (b) Cumulative distribution of diversity gain predictions at different elevation angle (USP).

a good representation of cumulative distribution function of the measurement site as compared with other prediction models. The model can thereby be employed in rain attenuation prediction for tropical countries with similar rain rate patterns to that of Malaysia.

Also, a new empirical site diversity prediction model is derived using rain attenuation measurements and TRMM radar data from five countries in Southeast Asia. The improvement on the model is the inclusion of low elevation angles and a high link frequency up to $70 \mathrm{GHz}$. The performance validation of the new prediction model is tested using three existing SD models over different link parameters. The comparison results with Hodge, Panagopoulos and Nagaraja empirical prediction show that the proposed model provides a better performance for site separation distance and elevation angle.

The overall results show that the last two models (Panagopoulos and Nagaraja) are not suitable for prediction
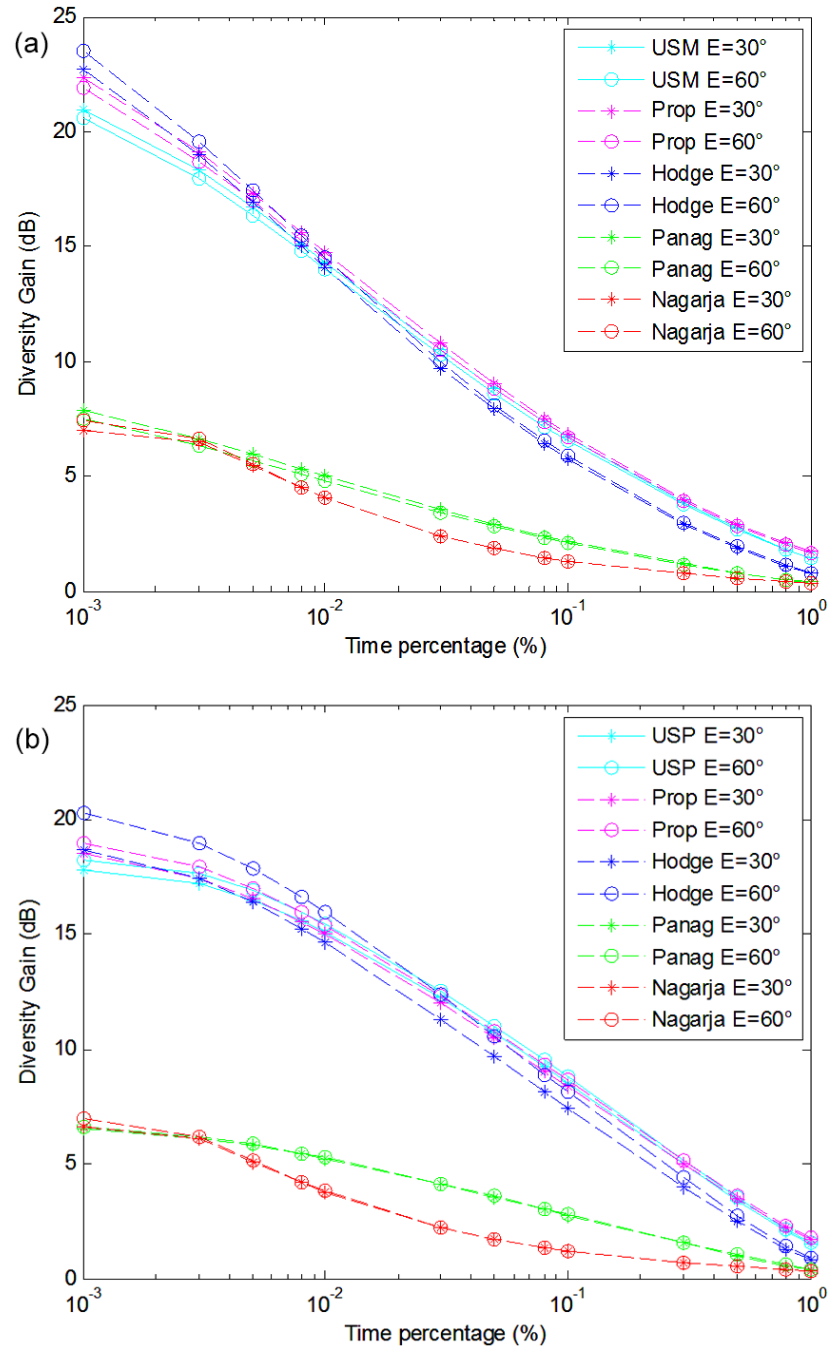

Figure 11. (a) Cumulative distribution of diversity gain predictions at different baseline angle (USM). (b) Cumulative distribution of diversity gain predictions at different baseline angle (USP).

of site diversity gain in tropical regions. Although the models tested by the authors proved to perform better than the Hodge model, their performance has been found unsuitable for tropical regions. On the side of the Hodge model, the performance is still relatively acceptable as compared to the other two models. It can be employed at high altitude in tropical and temperate regions. Its applicability still needs to be tested with more in situ SD measurements for further clarification. Above all, the performance of the proposed site diversity model has been found to be very good, and the percentage error is within the allowable error limit as determined in the ITU-R 618 recommendation.

Acknowledgements. The authors would like to thank Universiti Sains Malaysia, Universiti Kebangsaan Malaysia and Ladoke Akintola University of Technology for their support. 
Topical Editor V. Kotroni thanks the two anonymous referees for their help in evaluating this paper.

\section{References}

Bosisio, A. V. and Riva, C.: A Novel Method for the Statistical Prediction of Rain Attenuation in Site Diversity System: Theory and Comparative Testing against Experimental data, Int. J. Satell. Commun. Network, 16, 47-52, 1998.

Callaghan, S. A., Boyes, B., Waight, A., Couchman, J., Walden, C. J., and Ventouras, S.: An investigation of site diversity and comparison with ITU-R recommendations, Radio Sci., 43, 1-8, 2008.

Capsoni, C. and Matricciani, E.: Orbital and site diversity systems in rain environment: Radar-derived results, IEEE Trans. Antennas Propagat., AP-33, 517-522, 1985.

Castanet, L., Lemorton, J., and Bousquet, M.: Fade mitigation techniques for services at Ku-band and above: A review, Proc. 4th Ka-Band Utilization Conf., 2-4 November 1998, Venice, Italy, 1998.

Crane, R. K. and Shieh, H. C.: A two component rain model for the prediction of site diversity performance, Radio Sci., 24, 641-665, 1989.

Dissanayake, A. W., Allnutt, J. E., and Haidara, F.: A prediction model that combines rain attenuation and other propagation impairments along Earth-satellite paths, IEEE Transactions on Antenna and Propagation, 45, 1546-1557, 1997.

ESA: Radiowave propagation modeling for satcom services at $\mathrm{Ku}$ band and above, in: Final Report, ESA Publication Division, 2002.

Garcia, N. A. P. and da Silvo Mello, L. A. R.: Improved method for prediction of rain attenuation in terrestrial links, Electronic Lett., 40, 683-684, 2004.

Goddard, J. W. F. and Thurai, M.: Radar-Derived Path Reduction Factors for Terrestrial Systems, IEE 10th International Conference on Antenna and Propagation, 14-15 April 1997, Conference Publication No. 436, 2.218-2.221, 1997.

Hodge, D. B.: An improved model for diversity gain on earth-space propagation paths, Radio Sci., 17, 1393-1399, 1982.

Ippolito, L. J.: Satellite Communication systems engineering: atmospheric effects, Satellite link design, and system performance, John Wiley and Sons Ltd., USA, 2008.

ITU-R: Utilization of fade countermeasure strategies and techniques in the fixed-satellite service, Recommend. 1061 ITU-R S Ser., Int. Telecommun., Union, Geneva, 1994.

ITU-R: Propagation data and prediction methods Requirement for the design of Earth-space, Recommend. 618-10 ITU-R P Ser., Int. Telecommun. Union, Geneva, 2009a.
ITU-R: Acquisition, presentation and analysis of data in studies of tropospheric propagation, Recommend. 311-13 ITU-R P Ser., Int. Telecommun., Union, Geneva, $2009 \mathrm{~b}$.

Luini, L., Jeannin, N., Capsoni, C., Paraboni, A., Riva, C., Castanet, L. and Lemorton, J.: Weather Radar Data for Site Diversity Prediction and Evaluation of the Impact of Rain Field Advection, Int. J. Satell. Commun. Network, 29, 79-96, 2011.

Mandeep, J. S. and Allnutt, J. E.: Rain attenuation predictions at ku-band in south east asia countries, PIER, 76, 65-74, 2007.

Mandeep S. J. S., Hassan, S. I. S., Ain, M. F., Ghani, F., Kiyoshi, I., Kenji, T., and Mitsuyoshi, I.: Earth-To-Space Improved Model for Rain Attenuation Prediction at Ku-Band, Am. J. Appl. Sci., 3, 1967-1969, 2006.

Marshall, J. S. and Palmer, W. M. K.: The Distribution of Raindrops with Size, J. Meteorol., 5, 165-166, 1948.

Mass, J.: A simulation study of rain attenuation and diversity effects on satellite links, COMSAT Tech Rev., 17, 159-187, 1987.

Matricciani, E.: Prediction of site diversity performance in satellite communications systems affected by rain attenuation: Extension of the two layer rain model, Eur. Trans. Telecommun., 5, 327336, 1994.

Moupfouma, F.: Improvement of rain attenuation prediction method for terrestrial microwave links, IEEE Trans. Antennas Propag., 32, 1368-1372, 1984.

Nagaraja, C. and Otung, I. E.: Statistical Prediction of Site Diversity Gain on Earth-Space Paths Based on Radar Measurements in the UK, IEEE Trans. Antennas Propagat., 60, 247-256, 2012.

Pan, Q. W., Allnutt, J. E., and Tsui, C.: Evaluation of diversity and power control techniques for satellite communication systems in tropical and equatorial rain climates, IEEE Trans. Antennas Propag., 56, 3293-3301, 2008.

Panagopoulos, A. D., Arapoglou, P. D. M., and Cottis, P. G.: Satellite communications at $\mathrm{Ku}, \mathrm{Ka}$, and $\mathrm{V}$ bands: Propagation impairments and mitigation techniques, IEEE Commun. Surveys, 6, 2-14, 2004.

Panagopoulos, A. D., Arapoglou, P. D. M., Kanellopoulos, J. D., and Cottis, P. G.: Long-Term Rain Attenuation Probability and Site Diversity Gain Prediction Formulas, IEEE Transactions on Antennas and Propagation, 53, 2307-2313, 2005.

Yeo, J. X., Lee, Y. H., and Ong, J. T.: Performance of Site Diversity Investigated Through RADAR Derived Results, IEEE Transactions on Antennas and Propagation, 59, 3890-3898, 2011. 